\title{
Communication
}

\section{Incomplete-Leaf Multilevel Fast Multipole Algorithm for Multiscale Penetrable Objects Formulated With Volume Integral Equations}

\author{
Manouchehr Takrimi, Özgür Ergül, and Vakur B. Ertürk
}

\begin{abstract}
Recently introduced incomplete-leaf (IL) tree structures for multilevel fast multipole algorithm (referred to as IL-MLFMA) is proposed for the analysis of multiscale inhomogeneous penetrable objects, in which there are multiple orders of magnitude differences among the mesh sizes. Considering a maximum Schaubert-Wilton-Glisson function population threshold per box, only overcrowded boxes are recursively divided into proper smaller boxes, leading to IL tree structures consisting of variable box sizes. Such an approach: 1) significantly reduces the CPU time for near-field calculations regarding overcrowded boxes, resulting a superior efficiency in comparison with the conventional MLFMA where fixed-size boxes are used and 2) effectively reduces the computational error of the conventional MLFMA for multiscale problems, where the protrusion of the basis/testing functions from their respective boxes dramatically impairs the validity of the addition theorem. Moreover, because IL-MLFMA is able to use deep levels safely and without compromising the accuracy, the memory consumption is significantly reduced compared with that of the conventional MLFMA. Several examples are provided to assess the accuracy and the efficiency of IL-MLFMA for multiscale penetrable objects.
\end{abstract}

Index Terms-Incomplete leaf (IL), multilevel fast multipole algorithm (MLFMA), multiscale problems, volume integral equations (VIEs).

\section{INTRODUCTION}

Frequency-domain solutions of surface integral equations (SIEs) and volume integral equations (VIEs) via the method of moments (MoM) [1] and its accelerated versions, such as the fast multipole method (FMM) [2] and the multilevel fast multipole algorithm (MLFMA) [3], are among the most promising choices for multiscale problems [4], and almost all of the possible error sources commonly encountered in these solvers are well documented in the literature [5], [6]. However, when multiscale electromagnetic problems regarding penetrable objects that possess fine structural details, which require more dense local discretizations, are concerned, a hidden source of error contaminates the total process of the solution. Such an error, as detailed in [7], originates from the fact that most of the aforementioned methods deploy fixed-size boxes in conjunction with commonly approved "one-buffer-box" scenario [5] to carry out the far-field interactions within the leaf level. Note that accurate results may be obtained with fixed-size boxes by using fine meshes over or inside the entire geometry to comply with higher

Manuscript received June 30, 2016; revised April 5, 2017; accepted June 4, 2017. Date of publication July 3, 2017; date of current version September 1, 2017. This work was supported in part by the Islamic Azad University of Urmia under Grant 24289. (Corresponding author: Vakur B. Ertürk.)

M. Takrimi was with the Department of Electrical and Electronics Engineering, Bilkent University, TR-06800 Ankara, Turkey. He is now with the Department of Electrical Engineering, Islamic Azad University, Urmia Branch, Urmia 57169-63896, Iran (e-mail: m.takrimi@iaurmia.ac.ir).

Ö. Ergül is with the Department of Electrical and Electronics Engineering, Middle East Technical University, TR-06800 Ankara, Turkey.

V. B. Ertürk is with the Department of Electrical and Electronics Engineering, Bilkent University, TR-06800 Ankara, Turkey (e-mail: vakur@ee.bilkent.edu.tr).

Color versions of one or more of the figures in this communication are available online at http://ieeexplore.ieee.org.

Digital Object Identifier 10.1109/TAP.2017.2722858 discretization density required at more dense regions of the object. However, such an attempt ends up with a huge number of unknowns requiring excessive amount of memory in addition to being extremely inefficient even with a large number of levels for the conventional MoM-based solvers including MLFMA.

Although some new methods [8], [9] have been successfully demonstrated to reduce the memory and computational costs of MoM to $\mathcal{O}(N)$ for almost uniform discretizations, when the object possesses fine features and needs to be discretized with multisized meshes, most of those fast algorithms break down or at least lose efficiency and must be modified [4], [10] in order to reduce the overall computational complexity.

In this communication, the concept of incomplete-leaf (IL) tree structures (and hence, IL-MLFMA), which is introduced for SIEs in [7], is extended to VIEs, where we deploy Schaubert-WiltonGlisson (SWG) [11] functions to discretize the electric-field VIE based on the D-formulation [12] to solve electromagnetic scattering from multiscale, inhomogeneous, penetrable objects with arbitrary shapes. For such scatterers discretized with tetrahedral elements, only the overcrowded boxes in which the SWG populations exceed a predetermined threshold are recursively bisected into smaller boxes with other boxes remaining intact leading to IL tree structures. As a result, IL tree structures comprise variable box sizes that are distributed across the whole tree structure and not at the leaf level only. Hence, the error in the conventional MLFMA for multiscale problems due to the protrusion of basis/testing functions from their respective boxes, which impairs the validity of the addition theorem [5] used for farzone interactions, is reduced. This fact enables IL-MLFMA to use deep levels very safely without compromising the accuracy. Consequently, for VIEs, in comparison with the conventional MLFMA where fixed-size boxes are used: 1) the CPU time for near-field calculations of overcrowded boxes is considerably reduced leading to a superior efficiency and 2) the memory consumption is significantly reduced, which has not been the case for SIEs. It should be noted, at this point, that in the IL-MLFMA, the box sizes at the leaf level may be electrically very small (low-frequency regime). Interestingly, VIEs seem to be unaffected by the low-frequency breakdown as discussed in [13], and hence, using very deep levels of IL-tree structure is quite safe without any numerical instability. Finally, as in the case of SIEs, IL-MLFMA for VIEs can be merged with other methods in a similar fashion to the conventional MLFMA. We also note that a nonuniform oct-tree concept, which resembles the IL tree structures presented in this communication, has been proposed in [14], which develops a hybrid scheme by combining the accelerated Cartesian expansion algorithm with FMM for multiscale problems.

This communication is organized as follows. Section II presents a comprehensive description of the error originating from multiscale discretization, followed by an exposition regarding the IL tree structure. Simulation results demonstrating the numerical stability, accuracy, and efficiency of IL-MLFMA on some canonical and reallife problems are given in Section III. Section IV presents the memory 


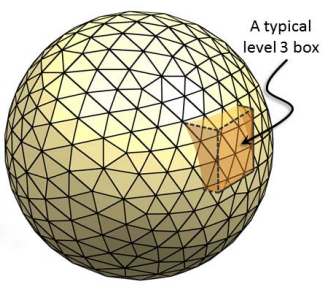

(a)

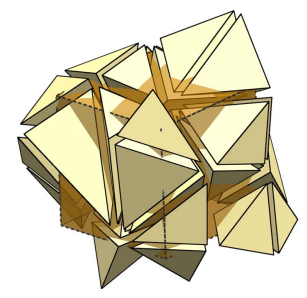

(b)

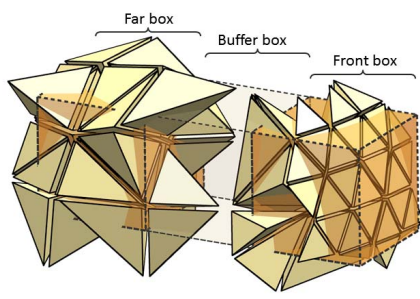

(c)

Fig. 1. Protrusions inside typical volume discretizations. (a) Sphere of $R=\lambda$ discretized with a mesh size of $\lambda / 10$ and a typical level 3 box located at the front of the object. (b) Distribution of tetrahedrons inside one of the internal boxes at level 3 when the multiscale factor is 4 . (c) Distribution of tetrahedrons within three consecutive boxes with a buffer box in between when the multiscale factor is 4 . Note that the front box is the same box as shown in (a).

performance of IL-MLFMA, followed by our concluding remarks in Section V.

\section{Multiscale Mesh as a Source of Error}

We investigate one of the leaf-level boxes in the context of MLFMA tree structure to visualize possible hidden errors imposed by multiscale volume discretizations. By defining "multiscale factor" as the ratio of the largest edge length to the smallest one within the entire discretized region, Fig. 1(a) shows a sphere of $R=\lambda$ discretized with a mesh size of $\lambda / 10$ and a multiscale factor of 4 . After recursively clustering the object up to level 3 (i.e., FMM level), we choose one of the leaf-level boxes inside the sphere and close to the origin. Fig. 1(b) demonstrates the mentioned box (with dashed edges) and includes all of 33 tetrahedrons that belong to this box. Note that these tetrahedrons have been shrunk by $20 \%$ in order to discriminate their relative positions throughout the transparent box. A careful probing reveals that more than 22 of tetrahedrons have a slight or even drastic protrusion, penetrating inside the nearby boxes. Apparently, this is not a matter of concern for those touching boxes where the interactions are computed based on MoM. However, regarding far-field interactions, it is an important source of error. Fig. 1(c) shows three consecutive boxes starting from the same front box shown in Fig. 1(a), where most of the belonging SWG functions are depicted here without showing other tetrahedrons. Since in the conventional MLFMA the mutual interaction between two end boxes is carried out by invoking the addition theorem, it is clearly seen that many of those tetrahedrons are very close to each other and hence, violating the necessary conditions to use conventional farzone computations. This is mainly due to the use of fixed-size boxes in all of the conventional FMM-based tree structures in which the leaf-level boxes are not capable of enclosing some or many of the larger discretized elements any more. This phenomenon also happens for SIEs, as detailed in [7], but it is observed to be more severe for volume discretizations and higher multiscale factors.

Careful observations reveal that while for reasonable meshes with a low multiscale factor (e.g., less than 8) only far interactions are seriously affected in terms of accuracy, as the multiscale factor increases (e.g., more than 50), very far interactions within a level, and even far-zone interactions across the levels are prone to a considerable error. As a brute-force remedy, increasing the box size effectively diminishes the error, but it also dramatically increases the computation time due to $\mathcal{O}\left(N^{2}\right)$ complexity regarding self-field and near-field interactions.

Note that the definition of near- and far-box concepts, the rules to determine the list of near and far boxes, and the essence of the related algorithms for VIEs are similar to those presented in [7]. Furthermore, the IL tree structure can be reduced to a traditional one (by forcing the program to equally continue the clustering operation starting from the second level by assigning a unity population threshold for all higher levels except the last leaf level). In this case, the conventional MLFMA is recovered, though some of the last level boxes may be overcrowded.

\section{NumERICAL RESUlTS}

The conventional MLFMA is a level-based algorithm. However, for IL-MLFMA, the required number of levels is automatically determined based on the maximum box population (MBP) per box. It should be mentioned that for a wide range of MBP values (except for very small values), the iterative solver converges rapidly and the error is almost constant. This unique feature provides us to optimize the total efficiency of IL-MLFMA as a function of MBP values, and these optimal values for MBP in VIEs are similar to those reported for SIEs [7]. Nevertheless, the efficiency and accuracy comparisons of IL-MLFMA are presented based on the number of levels as it is the only common parameter between the conventional MLFMA and IL-MLFMA.

In all numerical results, the relative root-mean-square (rms) error given by

$$
\text { Relative RMS Error }=\sqrt{\frac{\sum_{i=1}^{N}\left|\boldsymbol{E}_{\theta_{i}}^{\mathrm{Sim}}-\boldsymbol{E}_{\theta_{i}}^{\mathrm{Ref}}\right|^{2}}{\sum_{i=1}^{N}\left|\boldsymbol{E}_{\theta_{i}}^{\mathrm{Ref}}\right|^{2}}}
$$

is used to assess the accuracy of the proposed IL-MLFMA for VIEs. In (1), $\boldsymbol{E}_{\theta_{i}}^{\text {Sim }}$ and $\boldsymbol{E}_{\theta_{i}}^{\text {Ref }}$ are the dominant $\theta$ components of the far-zone electric field, obtained from IL-MLFMA (or conventional MLFMA) and the reference solution (Mie-series solutions for canonical sphere problems and MoM solutions for complicated geometries whose analytic solutions are not available), respectively. In all simulations, the generalized minimal residual iterative solver is used with an error tolerance of $10^{-3}$ and a restart value of 1000 without any preconditioner. Besides, the second-order Gaussian quadrature is used in the integrations in the volumes of the tetrahedral elements.

\section{A. Canonical Sphere Problems}

The first set of numerical results is canonical sphere problems (single or multilayer), whose analytic Mie-series solutions are known. Fig. 2 (inset) shows a homogeneous dielectric sphere of radius $R=60 \mathrm{~mm}(R \approx \lambda / 4)$ and $\epsilon_{r}=4$, illuminated by a $\hat{x}$-polarized uniform plane wave at $600 \mathrm{MHz}$, propagating in the $z$-direction. The sphere is discretized with a highly nonuniform volumetric mesh consisting of 49452 tetrahedrons (105081 SWG functions), where the edge sizes vary from $0.1 \mathrm{~mm}(\lambda / 2500)$ at the north pole up to $25 \mathrm{~mm}(\lambda / 10)$ at the south pole yielding a multiscale factor of 250 . By assigning four different values for MBP from 50 to 1500 (regarding IL-MLFMA) and four consecutive levels from 3 to 6 (regarding conventional MLFMA), Fig. 2 shows the total run time [right vertical axis and the solid (red) lines] and the relative rms error [left vertical 


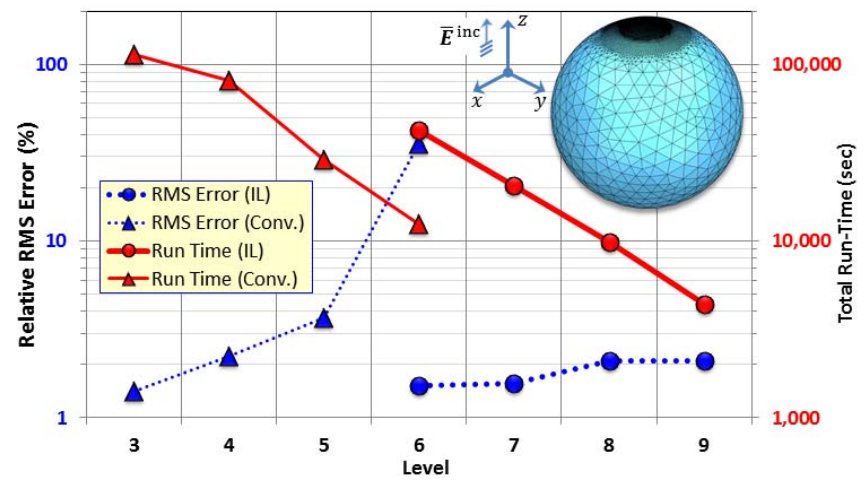

Fig. 2. Total run times and relative rms errors versus the common number of levels for both versions of MLFMA at $f=600 \mathrm{MHz}$ for a homogeneous dielectric sphere with $R=60 \mathrm{~mm}, \epsilon_{r}=4$, and a multiscale factor of 250 . The mesh size varies from 0.1 up to $25 \mathrm{~mm}$, consisting of 49452 tetrahedrons and 105081 SWG functions. Black (blue) dotted lines and gray (red) solid lines along with the corresponding logarithmic vertical axis at left and right show relative rms errors and total run times, respectively.

axis and the dotted (blue) lines] versus the number of levels for both versions of MLFMA. Note that both axes are in logarithmic scale and the relative rms error is calculated using the Mie-series solution as a reference.

Regarding the accuracy, both MLFMA solutions are comparable to each other up to level 4 for an error $<2 \%$. However, due to a high multiscale factor, conventional MLFMA starts to become inaccurate after level 4 (with 2\% error) and it fails to converge beyond level 6 (with $35 \%$ error). The error increases dramatically from $1.5 \%$ (FMM level) up to $35 \%$ (for level 6). On the other hand, as shown in Fig. 2, IL-MLFMA always converges for a wide range of MBPs spanning three orders of magnitude (i.e., from 25000 down to 25 ; equivalently from MoM level to level 10) and the error remains almost stable with only $0.5 \%$ increase from level $6(\mathrm{MBP}=1500)$ up to level 9 $(\mathrm{MBP}=50)$. A slight increase in the error for very small MBP values is due to some protrusions for very small boxes as expected.

Regarding the efficiency, IL-MLFMA demonstrates much better efficiency for some optimal values of MBP (i.e., from 120 to 50 equivalent with the eighth or ninth level). Accepting the error margin as less than $2 \%$, the best timing for the conventional MLFMA belongs to the fourth level with $81000 \mathrm{~s}$, whereas it is $4400 \mathrm{~s}$ for IL-MLFMA corresponding to the ninth level.

A two-layer sphere in Fig. 3 (inset) with the inner and outer radii of $20 \mathrm{~mm}\left(\epsilon_{r}=16\right)$ and $200 \mathrm{~mm}\left(\epsilon_{r}=2\right)$, respectively, forms the next example. After discretization, the edge sizes vary from 1 to $75 \mathrm{~mm}$ (multiscale factor $=75$ ) with 33450 tetrahedrons (71594 SWG functions). Due to different permittivities, the fine meshed regions mostly belong to the core of the sphere while the coarsely meshed regions are embedded inside the cladding. Illuminating the sphere with a similar uniform plane wave at $800 \mathrm{MHz}$, Fig. 3 shows the far-zone scattered electric-field values versus the bistatic angle for IL-MLFMA with $\mathrm{MBP}=100$, conventional MLFMA for levels 4 up to 6 (no other solutions for higher levels due to convergence problems), and the Mie-series solution. The accuracy for the fourth and the fifth levels of the conventional MLFMA is comparable with the accuracy of IL-MLFMA, as shown in Table I, where the provided errors are mostly due to discretization (more dominant in lower levels than the error incurred by the protrusions). However, the six-level conventional MLFMA yields a higher error, mostly dominated by the protrusions, and as we go deeper levels (after level 6), convergence problems occur. Table I also provides the total run times, which show that IL-MLFMA is significantly more efficient than the conventional one.

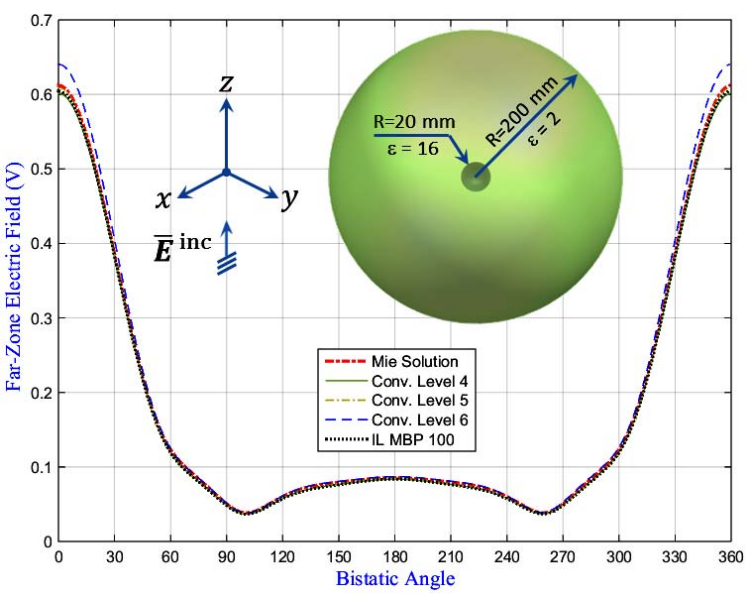

Fig. 3. Far-zone electric field scattered from a two-layer dielectric sphere with the inner radius of $R=20 \mathrm{~mm}\left(\epsilon_{r}=16\right)$ and the outer radius of $R=200 \mathrm{~mm}$ $\left(\epsilon_{r}=2\right)$. The sphere is illuminated by a unit amplitude uniform plane wave at $f=800 \mathrm{MHz}$. The results from the Mie-series solution and from both MLFMA versions are presented for comparison.

TABLE I

Performance Comparison Between IL-MLFMA AND CONVENtional MLFMA Applied to the Double Layer Sphere Shown IN Fig. 3 at $f=800$ MHz With a Multiscale Factor of 75

\begin{tabular}{l|c|c} 
& Total Run-Time & Rel. RMS Error \\
\hline Conv. (Level 4) & $95,131 \mathrm{~s}$ & $3.26 \%$ \\
\hline Conv. (Level 5) & $46,316 \mathrm{~s}$ & $3.55 \%$ \\
\hline Conv. (Level 6) & $31,900 \mathrm{~s}$ & $4.88 \%$ \\
\hline IL (MBP 100) & $12,690 \mathrm{~s}$ & $3.66 \%$ \\
\hline
\end{tabular}

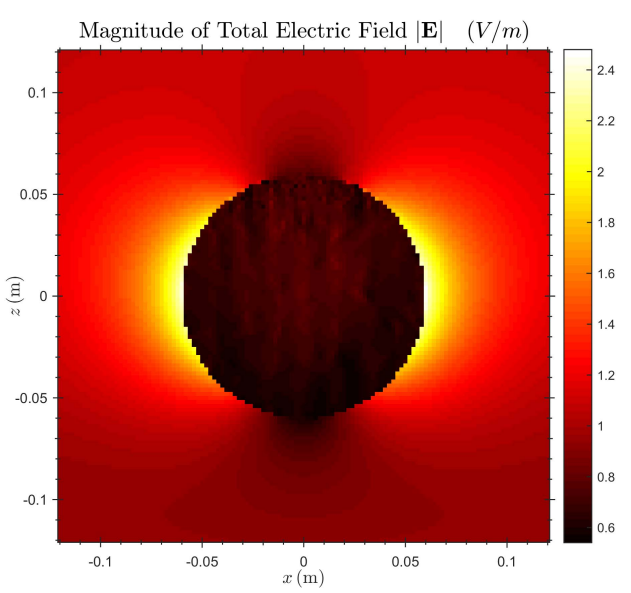

Fig. 4. Near-field total electric field values on a symmetrical $\lambda \times \lambda \mathrm{m}^{2}$ rectangular area residing on the $x z$ plane for the dielectric sphere shown in Fig. 2 (inset) illuminated by a $\hat{x}$-polarized uniform plane wave at $600 \mathrm{MHz}$, propagating in the $z$-direction.

\section{B. Near-Field Assessment}

To evaluate the accuracy of IL-MLFMA for near-field computations, the same homogeneous dielectric sphere shown in Fig. 2 (inset) is used. Fig. 4 shows the magnitude of the total electric field inside and outside of the sphere within a $\lambda \times \lambda \mathrm{m}^{2}$ symmetrical rectangular area on the $x z$ plane. Invoking the near-field Mie-series solution as a reference and by using a similar error assessment strategy given in (1) for all three components, the near-field relative rms errors are $2.1 \%$ for IL-MLFMA (equivalent to level 8 or 9) and $1.85 \%, 3.52 \%$, and $293 \%$ for levels $4-6$ of the conventional MLFMA, respectively. 


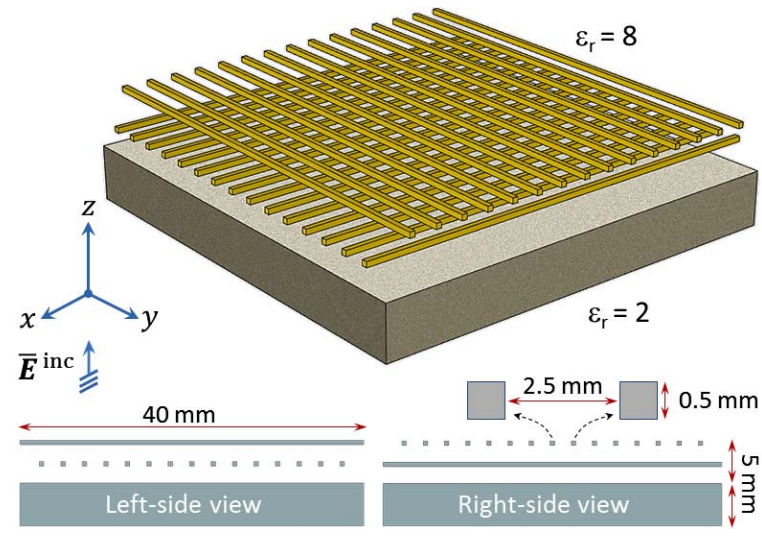

Fig. 5. Dielectric resonance structure consisting of three layers. The bottom layer is a slab of $40 \times 40 \times 5 \mathrm{~mm}^{3}$ with $\epsilon_{r}=2$, while two other top layers contain two sets of 15 parallel dielectric rods with $\epsilon_{r}=8$ perpendicular to each other. All dimensions and side views are shown.

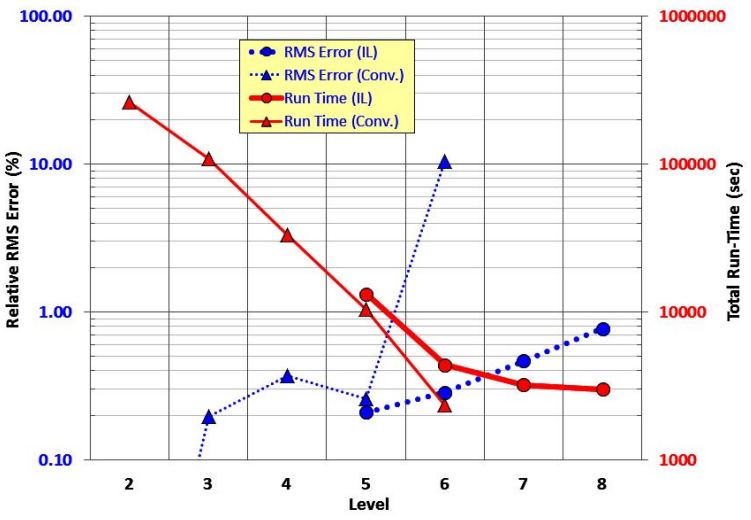

Fig. 6. Total run times and relative rms errors versus the common number of levels for both versions of MLFMA, where the geometry shown in Fig. 5 is used at $f=2 \mathrm{GHz}$. Circle and triangle markers are used to separate IL-MLFMA and the conventional MLFMA, respectively. Black (blue) dotted lines and gray (red) solid lines along with the corresponding logarithmic vertical axis at left and right show relative rms errors and total run times, respectively.

These results clearly demonstrate the superior accuracy of IL-MLFMA in comparison with the conventional MLFMA.

\section{Complicated Objects}

A fairly complicated dielectric resonance structure consisting of three layers is shown in Fig. 5. The bottom layer is a $40 \times 40 \mathrm{~mm}^{2}$ dielectric slab of thickness $5 \mathrm{~mm}$ with $\epsilon_{r}=2$ and is loaded by two groups of perpendicular arrays at the top of each other with 2.5-mm spacing, where each group contains 15 parallel dielectric rods with $\epsilon_{r}=8$. The left- and right-side views together with all other dimensions are given in Fig. 5. The bottom layer is discretized with an average mesh size of $5 \mathrm{~mm}$, while the dielectric bars are discretized with an average mesh size of $0.5 \mathrm{~mm}$, leading to 37253 tetrahedrons and a multiscale factor of 22 .

This structure is illuminated by the same plane wave as used for the previous examples at $f=2 \mathrm{GHz}$. The far-zone electric fields are computed with both MLFMA versions and compared with MoM solution, which acts as a reference solution to assess the relative rms error. Fig. 6 shows the total run time [right logarithmic vertical axis and the solid (red) lines] and the relative rms error [left logarithmic vertical axis and the dotted (blue) lines] versus the number of levels

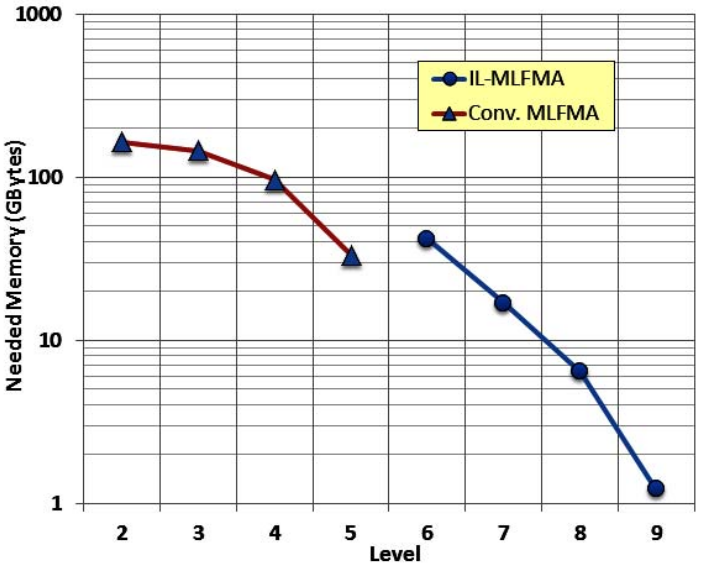

Fig. 7. Amount of the required memory to store the near-field interactions and also to compute the far-field interactions for the canonical scattering problem shown in Fig. 2.

for both versions of MLFMA. The best timing for the conventional MLFMA belongs to level 5 with approximately $10500 \mathrm{~s}$ and $0.25 \%$ error. However, for the same accuracy, the total timing is $4380 \mathrm{~s}$ for IL-MLFMA. Moreover, the total run time for IL-MLFMA is around $3000 \mathrm{~s}$ if under $0.8 \%$ error is acceptable. Although the conventional MLFMA is very accurate up to level 5 , the error increases 1.5 orders of magnitude starting from level 6. Considering the IL version, the error smoothly increases due to smaller MBP values. Note that there is an important difference between this example and the others regarding the discretization. For this structure, the leaf-level box boundaries from levels 3 up to 5 (equivalent to box sizes from 10 down to $2.5 \mathrm{~mm}$ ) are located in very special positions, as the resonance structure consists of flat and mostly parallel boundaries that are also parallel with the box boundaries. Besides, the structure is mostly discretized with right-angled tetrahedrons within the parallel rods, and some other highly ordered larger meshes inside the bottom layer. Therefore, very few number of tetrahedrons are getting cut by the box boundaries, leading to minimum possible protrusions regarding the SWG functions. Consequently, the conventional MLFMA performs very good up to level 5. But starting from level 6 (i.e., a box size of $1.25 \mathrm{~mm}$ ), almost all of the SWG functions are severely cut by the leaf-level box boundaries. Hence, heavy protrusions occur, and accuracy of the conventional MLFMA starts to decrease drastically.

\section{Memory Performance of IL-MLFMA}

In addition to the efficiency and accuracy of IL-MLFMA for multiscale penetrable objects, its storage requirements are significantly reduced compared with those of the conventional MLFMA, as IL-MLFMA is able to use the deeper levels safely without compromising the accuracy. To demonstrate this point, we reconsider the homogeneous sphere problem shown in Fig. 2 (inset). Recall from Fig. 2 that, for a relative rms error of less than $2 \%$, only the third and fourth levels of the conventional MLFMA may be used. For these levels, the required memory is 150 and $90 \mathrm{~GB}$, respectively, as seen in Fig. 7, which provides the needed memory (in GB) for both the conventional MLFMA and IL-MLFMA. Even for the fifth level (rms error is 3.5\%), the conventional MLFMA uses 32 GB of memory. However, for IL-MLFMA with a relative rms error of less than $2 \%$, the required memory is as low as $1.2 \mathrm{~GB}$ when $\mathrm{MBP}=50$ (equivalent to level 9).

Considering the more complicated dielectric structure shown in Fig. 5, a similar behavior regarding the memory consumption is 
TABLE II

Required Memory to Store the NeAR-Field Interactions and Also to COMPUTE THE FAR-Field INTERACTIONS For A DieleCTRIC STRUCTURE SHOWN IN FIg. 5 With THE RESULTS GIVEN IN Fig. 6

\begin{tabular}{c|c|c} 
& IL-MLFMA & Conv. MLFMA \\
\hline Level 2 & & $118 \mathrm{~GB}$ \\
\hline Level 3 & & $47.1 \mathrm{~GB}$ \\
\hline Level 4 & & $14.1 \mathrm{~GB}$ \\
\hline Level 5 & $5.1 \mathrm{~GB}$ & $3.9 \mathrm{~GB}$ \\
\hline Level 6 & $1.4 \mathrm{~GB}$ & \\
\hline Level 7 & $890 \mathrm{MB}$ & \\
\hline Level 8 & $650 \mathrm{MB}$ & \\
\hline
\end{tabular}

obtained, as given in Table II. It is seen that the minimum required memory within acceptable range of accuracy and efficiency for the conventional MLFMA is about 3.9 GB, while it reduces to $650 \mathrm{MB}$ when IL-MLFMA is used. A wide range of simulations in addition to above-mentioned examples reveal that, in larger problems, higher amount of memory can be saved due to the safe use of deeper levels.

\section{CONCLUSION}

The concept of IL structures (and hence, IL-MLFMA), originally introduced for SIEs, is extended to VIEs to analyze multiscale electromagnetic problems for inhomogeneous dielectric objects. As a result, by using a population-based nonuniform clustering for highly variable discretizations with large multiscale factors: 1) protrusions of SWG functions from the respective boxes are minimized that improve the accuracy; 2) the total number of interacting boxes is reduced that improves the efficiency; and 3) deeper levels, for particularly locally overmeshed regions, can be safely used that reduces the memory consumption significantly. Besides, as in the case of IL-MLFMA for SIEs, IL-MLFMA for VIEs also recovers the conventional MLFMA for uniform discretizations, and can be combined with other methods in a similar fashion to the conventional MLFMA.

\section{REFERENCES}

[1] R. F. Harrington, Field Computation by Moment Methods. New York, NY, USA: MacMillan, 1968

[2] R. Coifman, V. Rokhlin, and S. Wandzura, "The fast multipole method for the wave equation: A pedestrian prescription," IEEE Antennas Propag. Mag., vol. 35, no. 3, pp. 7-12, Jun. 1993.

[3] J. Song, C.-C. Lu, and W. C. Chew, "Multilevel fast multipole algorithm for electromagnetic scattering by large complex objects," IEEE Trans. Antennas Propag., vol. 45, no. 10, pp. 1488-1493, Oct. 1997.

[4] K. Yang and A. E. Yilmaz, "Integral equation solution of 3-D anisotropic lossy dielectrics in uniaxial layered media," in Proc. IEEE Antennas Propag. Soc. Int. Symp., Jul. 2012, pp. 1-2.

[5] W. C. Chew, E. Michielssen, J. M. Song, and J. M. Jin, Fast and Efficient Algorithms in Computational Electromagnetics. Boston, MA, USA: Artech House, 2001.

[6] S. Koc, J. Song, and W. C. Chew, "Error analysis for the numerical evaluation of the diagonal forms of the scalar spherical addition theorem," SIAM J. Numer. Anal., vol. 36, no. 3, pp. 906-921, Jan. 1999.

[7] M. Takrimi, Ö. Ergül, and V. B. Ertürk, "A novel broadband multilevel fast multipole algorithm with incomplete-leaf tree structures for multiscale electromagnetic problems," IEEE Trans. Antennas Propag., vol. 64, no. 6, pp. 2445-2456, Jun. 2016.

[8] A. D. Polimeridis, J. F. Villena, L. Daniel, and J. K. White, "Stable FFT-JVIE solvers for fast analysis of highly inhomogeneous dielectric objects," J. Comput. Phys., vol. 269, pp. 280-296, Mar. 2014.

[9] K. Yang, "FFT and multigrid accelerated integral equation solvers for multi-scale electromagnetic analysis in complex backgrounds," Ph.D. dissertation, Dept. Elect. Comput. Eng., Univ. Texas Austin, Austin, TX, USA, Aug. 2014.

[10] X. Millard and Q. H. Liu, "A fast volume integral equation solver for electromagnetic scattering from large inhomogeneous objects in planarly layered media," IEEE Trans. Antennas Propag., vol. 51, no. 9, pp. 2393-2401, Sep. 2003.

[11] D. H. Schaubert, D. R. Wilton, and A. W. Glisson, "A tetrahedral modeling method for electromagnetic scattering by arbitrarily shaped inhomogeneous dielectric bodies," IEEE Trans. Antennas Propag., vol. 32, no. 1 , pp. 77-85, Jan. 1984.

[12] M. M. Botha, "Solving the volume integral equations of electromagnetic scattering," J. Comput. Phys., vol. 218, no. 1, pp. 141-158, 2006.

[13] I.-T. Chiang and W.-C. Chew, "New formulation and iterative solution for low-frequency volume integral equation," J. Electromagn. Wave Appl., vol. 19, no. 3, pp. 289-306, 2005.

[14] M. Vikram, H. Huang, B. Shanker, and T. Van, "A novel wideband FMM for fast integral equation solution of multiscale problems in electromagnetics," IEEE Trans. Antennas Propag., vol. 57, no. 7, pp. 2094-2104, Jul. 2009. 